\title{
Analysis of Stress Distribution in Piezoelectric MEMS Energy Harvester Using Shaped Cantilever Structure
}

Jung-Hyun Park , Junsuk Kang , Hosang Ahn , Seon-Bae Kim , Dan Liu \& Dong-Joo Kim

To cite this article: Jung-Hyun Park , Junsuk Kang , Hosang Ahn , Seon-Bae Kim , Dan Liu \& Dong-Joo Kim (2010) Analysis of Stress Distribution in Piezoelectric MEMS Energy Harvester Using Shaped Cantilever Structure, Ferroelectrics, 409:1, 55-61, DOI: 10.1080/00150193.2010.487125

To link to this article: http://dx.doi.org/10.1080/00150193.2010.487125

曲 Published online: 01 Dec 2010.

Submit your article to this journal $₫$

Џلll Article views: 298

Q View related articles $๘$

4 Citing articles: 4 View citing articles 준 


\title{
Analysis of Stress Distribution in Piezoelectric MEMS Energy Harvester Using Shaped Cantilever Structure
}

\author{
JUNG-HYUN PARK, ${ }^{1}$ JUNSUK KANG,${ }^{2}$ HOSANG AHN, ${ }^{1}$ \\ SEON-BAE KIM, ${ }^{1}$ DAN LIU, ${ }^{1}$ AND DONG-JOO KIM ${ }^{1, *}$ \\ ${ }^{1}$ Materials Research and Education Center, Auburn University, AL 36849, USA \\ ${ }^{2}$ Department of Civil Engineering, Auburn University, AL 36849, USA
}

\begin{abstract}
Much interest in energy harvesters has been focused on maintaining their conversion efficiency during scaling down via the micromachining process. The piezoelectric PZTbased MEMS energy harvester was designed and fabricated to increase the fraction of the material strained during deflection since the geometric change of the cantilever shape can change the strain distribution on the beam and improve the output power. The generated power during beam deflection was separately collected from individual electrodes located at different positions of the cantilever, and they had good agreement with the strain estimation from finite element analysis. The trapezoidal shape showed $39 \%$ higher power than that of rectangular one.
\end{abstract}

Keywords Energy harvesting; MEMS; strain distribution; trapezoid

\section{Introduction}

There has been a growing interest in developing energy harvesters that generate electricity using a renewable energy source for very small and low power sensor nodes. Energy harvesters obtain the electricity converted from permanent and ambient environmental energy sources such as light, thermal, fluid flow, and mechanical vibration. Mechanical vibration has been considered as a promising source due to its wide permeation and efficient converting mechanism [1]. The vibration energy can be converted into electricity by electrostatic [2], electromagnetic [3], or piezoelectric transducers [4]. Among them, the piezoelectric transducer has become favorable to many researchers since it has high electromechanical coupling, simple configuration without external voltage source, and easy integration. The bulk prototypes based on bimorph or multimorph structures have been intensively studied, and a sufficient power level for wireless sensor nodes has been demonstrated $[5,6]$. Recently, studies of piezoelectric energy harvesters have focused on maintaining their conversion efficiency during scaling down via the micromachining process [7-10]. In our previous work, we demonstrated a successful microelectromechanical systems (MEMS) energy harvester using sol-gel deposited PZT, which generated $160 \mathrm{mV}$ and $3272 \mu \mathrm{Wcm}^{-3}$ at $19.6 \mathrm{~ms}^{-2}$ acceleration. However, such a device may not be implemented due to insufficient power output for current sensor devices, since piezoelectric film in a MEMS device is

Received August 23, 2009; in final form September 25, 2009.

${ }^{*}$ Corresponding author. E-mail: dkim@eng.auburn.edu 
commonly limited by single-layered unimorph structures and is clamped by massive substrate. In addition, the micromachining process can cause degradation of the piezoelectric properties of the thin films.

As a way to improve the output power in vibration energy harvesters, a cantilever design has been explored to change the strain distribution on piezoelectric films. The rectangular cantilever beam is commonly chosen due to simple design for microfabrication, but most of its stress is driven to near the anchor of the cantilever beam, and the converted power; therefore, it is dominated by the anchor area. In addition, such non-uniform distribution can weaken the strength of the cantilever structure during higher vibration conditions. Baker et al. have demonstrated that the trapezoidal shaped-cantilever increased the power output by as much as $30 \%$ compared with a rectangular cantilever in bulk scale [11]. Although much research has focused on investigating the strain distribution with the change of the cantilever shape, less attention has been paid to analysis and experimental verification of the devices in MEMS scale.

This study is designed to analyze the strain distribution on the cantilever geometry and verify such effects experimentally by measuring the output power locally from electrodes located along the cantilever beam. This analysis is applied to trapezoidal- and rectangularshaped cantilevers. Preliminary and experimental results are given to improve the power density by changing the strain distribution of a micromachined PZT cantilever.

\section{Fabrication and Experimental Characterization}

Figure 1 shows the rectangular- and trapezoidal-shaped cantilever energy harvesters fabricated by a micromachining technique. $\mathrm{Pb}\left(\mathrm{Zr}_{0.52} \mathrm{Ti}_{0.48}\right) \mathrm{O}_{3}$ thin film was deposited on $\mathrm{Pt} / \mathrm{Ti} / \mathrm{SiO}_{2} / \mathrm{Si}$ substrate by sol-gel process, and the thickness of PZT film was $1 \mu \mathrm{m}$. After patterning and micromachining cantilevers by dry etching using an Inductive Coupled plasma Deep Reactive Ion Etcher (ICP RIE, STS), the silicon proof mass and support layer were formed. The proof mass was designed to enhance the displacement under a given acceleration. The thickness of the silicon support layer was kept at $20 \mu \mathrm{m}$, which prevents
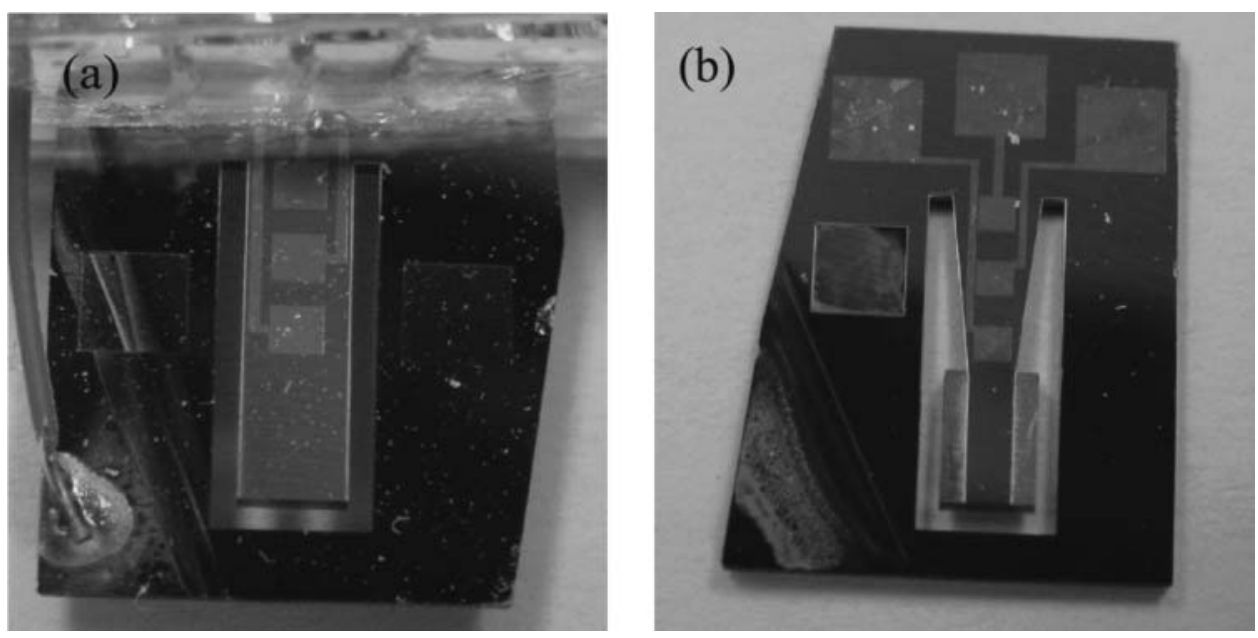

Figure 1. The photograph of rectangular and trapezoidal cantilevers: (a) rectangular; (b) trapezoidal. 


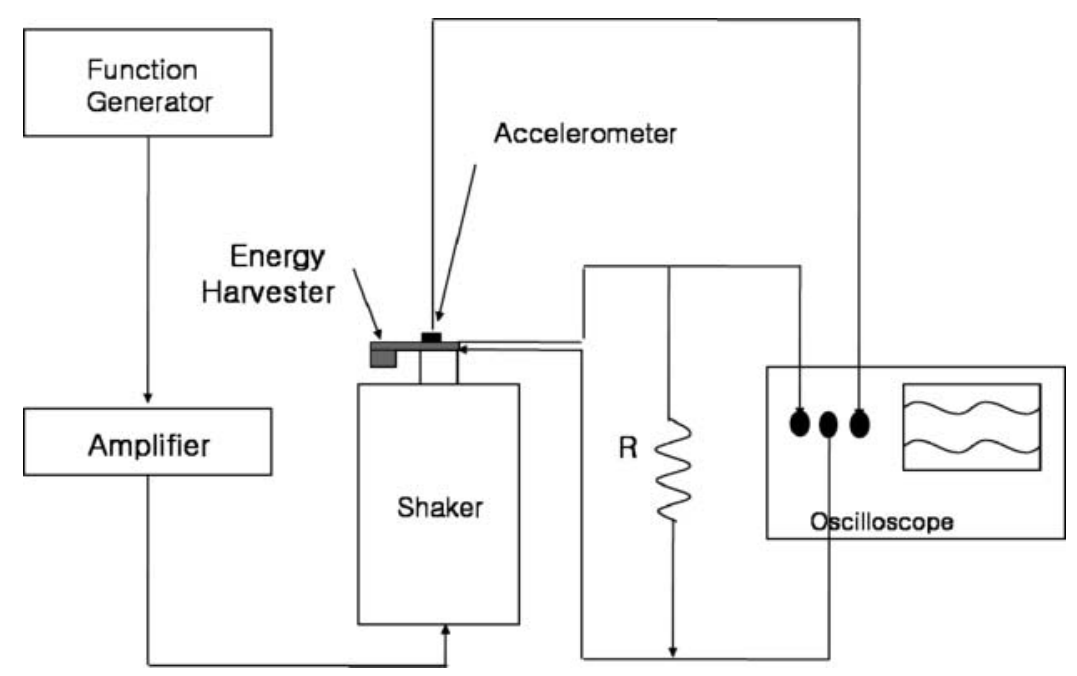

Figure 2. The illustration of experimental setup.

the bending of the cantilever structure after releasing the structure and provides mechanical strength at higher acceleration conditions. The detailed fabrication process of the device was published in our previous report [7]. The dimensions of both cantilevers were $4 \times 2$ $(\mathrm{L} \times \mathrm{W})$ for the beam and $3 \times 2 \times 0.5(\mathrm{~L} \times \mathrm{W} \times \mathrm{H})$ for the mass in the unit of $\mathrm{mm}$. The trapezoidal cantilever had a reduced width of $1 \mathrm{~mm}$ at the contact point with the proof mass.

Discrete platinum electrodes were separately patterned on each cantilever, i.e. near the anchor, middle and the mass, as shown in Fig. 1. Since the direct wire bonding might affect the vibration motion of a device and/or failure of wires during vibration, Pt thin films were patterned on a cantilever surface to extract signals from each electrode. As a wiring method on a contact pad, hand-made polymer packaging for a top electrode was performed and a silver paste was used to contact bottom electrodes.

Output signals were obtained from a device by using the experimental setup consisting of a function generator (Agilent 33220A), an amplifier, an electromagnetic shaker (Labworks ET-132-203), and an oscilloscope (Tektronix TDS3014B). The device was mounted on a shaker that provided a seismic vibration to the device at its resonant frequency. The acceleration of the generated vibration was monitored by an accelerometer (PCB Piezoelectronics 352C65) that was mounted on the shaker and connected to the oscilloscope. The generated current and voltage were measured by a resistor load. When the output power values were calculated by Ohm's law $P=V^{2} / R_{\text {load }}$, Root Mean Square (RMS) voltages were used to evaluate AC-DC conversion. The illustration of the setup is shown in Fig. 2. The resonant frequencies were measured by an impedance analyzer (Agilent Technologies, 4294A), and the values were $128.1 \mathrm{~Hz}$ and $117.3 \mathrm{~Hz}$ for rectangular and trapezoidal cantilevers, respectively. The resonant frequencies of the devices were designed to match ambient vibration sources $(60 \sim 200 \mathrm{~Hz})$ and showed a small discrepancy of less than $1 \%$ from the estimated values [7].

\section{FEM Analysis}

ABAQUS finite element modeling software was used to estimate the strain on the PZT layer. The modeling was performed by loading a static force of $9.8 \mathrm{~ms}^{-2}$ acceleration 
(a)

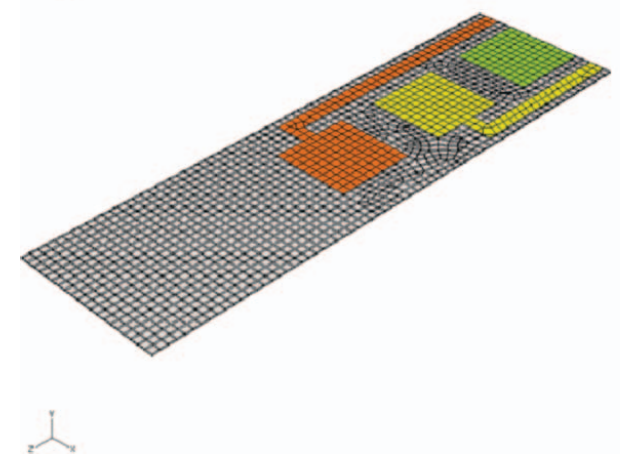

(b)

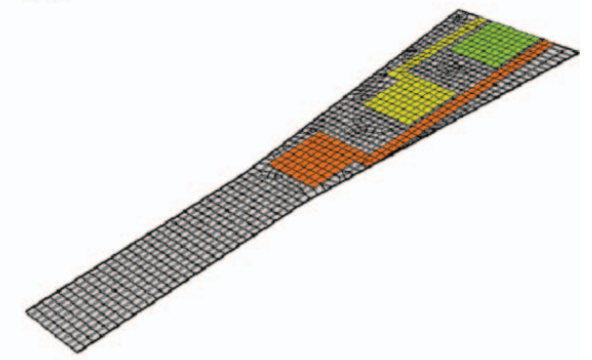

Figure 3. The configuration of electrode and mesh elements on (a) rectangular and (b) trapezoidal cantilevers. (See Color Plate V)

on the cantilever beam. The material properties used for the PZT layer were: a Young's modulus of $69 \mathrm{GPa}$ (measured by nano indentation); a Poisson's ratio of 0.3 ; and a density of $2330 \mathrm{Kgm}^{-3}$. The material properties used for Si were: a Young's modulus of $160 \mathrm{GPa}$ (110 direction); a Poisson's ratio of 0.27 ; and a density of $7500 \mathrm{Kgm}^{-3}$. The $\mathrm{SiO}_{2}$ layer was also considered in modeling and its properties were: a Young's modulus of $70 \mathrm{Gpa}$; a Posison's ratio of 0.165 ; and a density of $2200 \mathrm{Kgm}^{-3}$. The local strains at each electrode were obtained by averaging the strains of the mesh elements of the electrodes, as shown in Fig. 3.

\section{Results and Discussion}

The output power was measured at different electrodes for both cantilevers. Figure 4(a) and 4(b) show the normalized powers at a given resistive load for each electrode under $2.45 \mathrm{~ms}^{-2}$ of acceleration. The total power was normalized based on the real size of the actual electrode area of $1 \mathrm{~mm}^{2}$ after excluding the output power from a patterned wire electrode on a PZT layer. There were two limitations on this normalization. The first is that the generated charge on a piezoelectric material is not linearly proportional to the amount of strain due to the nonlinearity of PZT. The nonlinear piezoelectric coefficient, however, was neglected in this study. The other limitation is that the maximum power was shown at the optimal resistive loads of $20 \pm 1 \mathrm{k} \Omega$ of the device. Their small differences of the optimal resistive loads between electrodes resulted from the contribution of the patterned wire on a PZT layer to the output power. The maximum power obtained from each electrode was used to compare them with average strain values of each electrode for both cantilevers, as shown in Fig. 4(c) and 4(d). The electrodes near the anchor area showed the highest power (denote normalized power without notice from now on) in both cantilevers, while the output power at electrodes near the mass area had the smallest value. It is common that a large amount of strain occurs near the anchor and the strain is reduced near the mass. The output power from three electrodes showed a nonlinear relationship, indicating nonlinear strain distribution. The power at the middle electrode of the rectangular cantilever showed a smaller value compared to the average value calculated from the anchor and mass electrodes, while the trapezoidal cantilever showed a bit larger value. If it is assumed that the materials in a cantilever structure exhibit linear, elastic, and isotropic with an average 

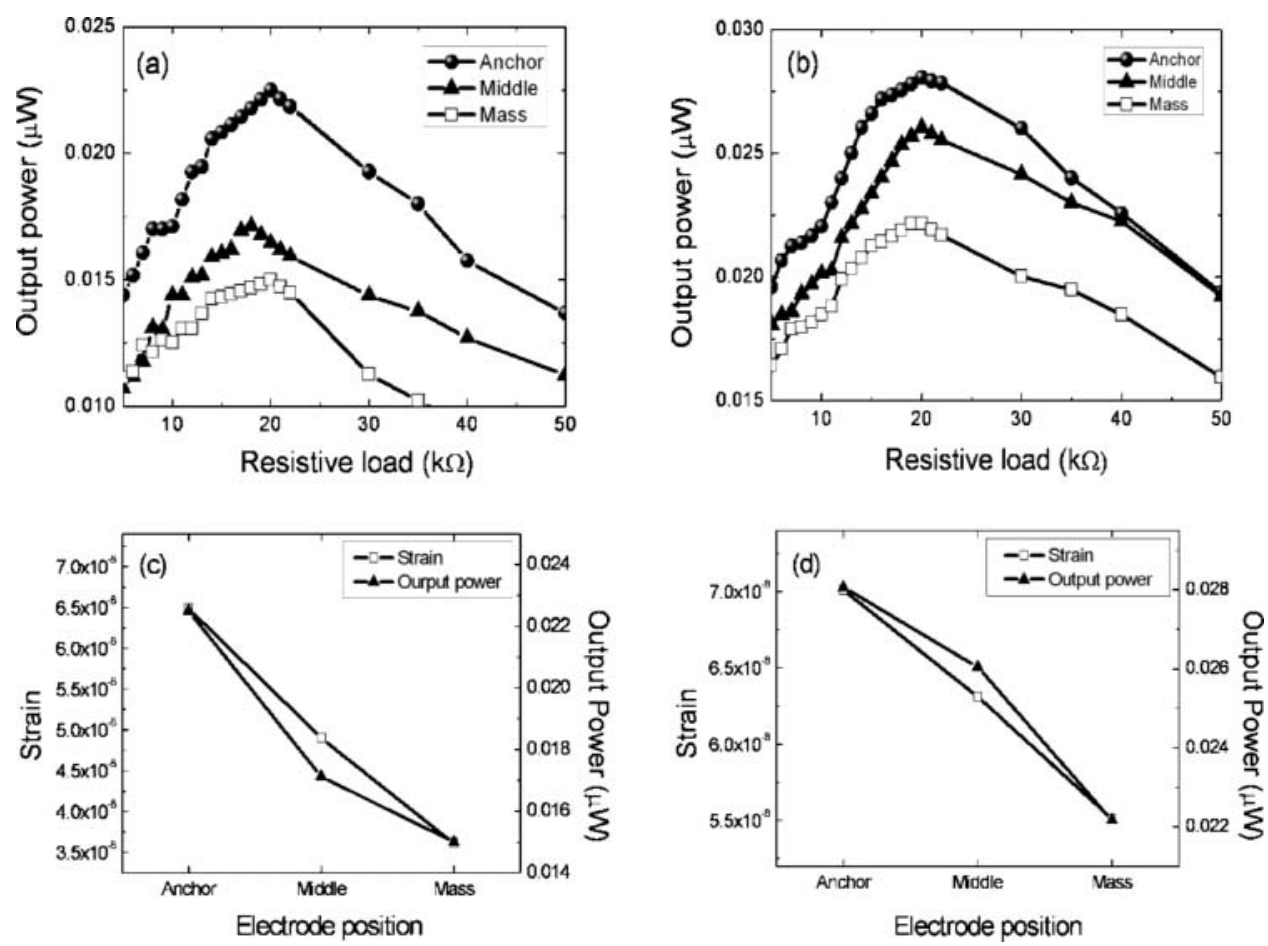

Figure 4. The output power from different electrodes for (a) a rectangular and (b) a trapezoidal cantilever with varying resistive load. Their maximum output power values were compared with the strains by FEM modeling for (c) the rectangular and (d) the trapezoidal cantilever.

stress applied along the 1-1 direction, the output power can be expressed as follows [12]:

$$
P=\frac{V^{2}}{R_{L}}=\frac{1}{R_{L}}\left\{g_{31} E \varepsilon\left(\frac{x}{L}\right) L\right\}^{2}
$$

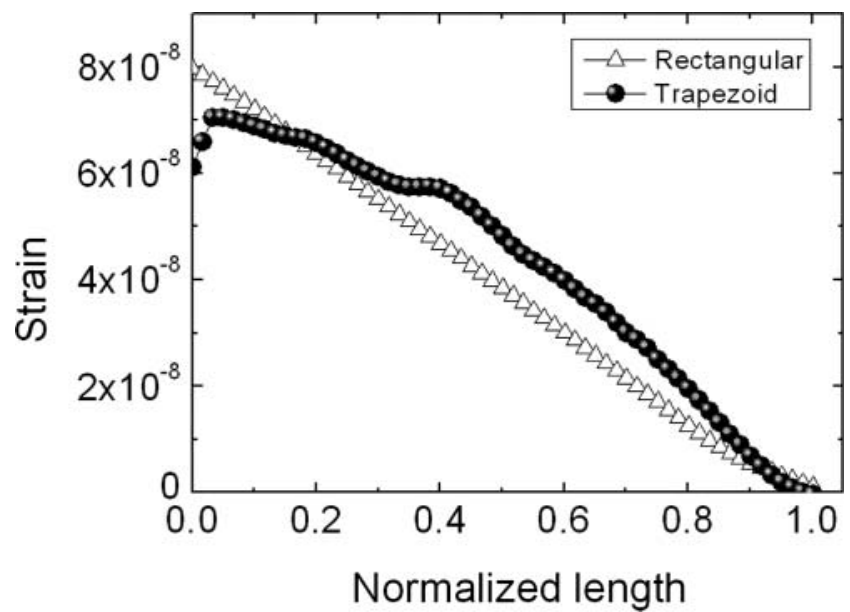

Figure 5. The strain profiles from anchor to mass for the rectangular- and trapezoid-shaped cantilevers. 


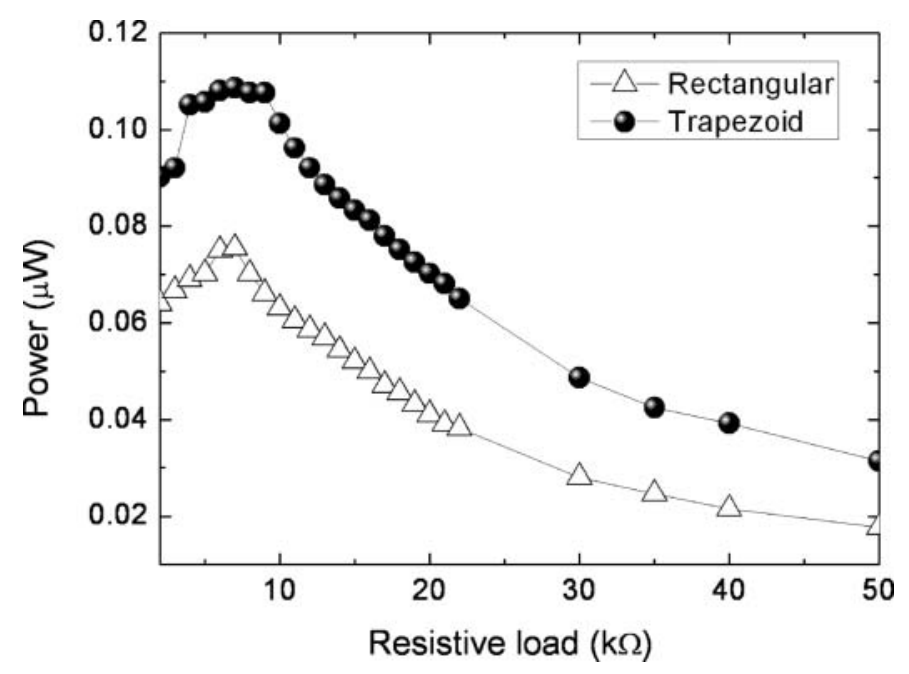

Figure 6. The generated total powers from rectangular- and trapezoid-shaped cantilevers.

Where $x$ is the location from the clamped end, $L$ is the length of the beam, $g$ is the piezoelectric voltage constant, $E$ is the Young's modulus, and $\varepsilon$ is the strain. In Fig. 4(c), the relationship between strain and power has good agreement with the equation (1). Conversely, the trapezoidal cantilever has a negative parabolic relationship. The reason is because the strain distribution has spread out into the region of the middle electrode in the trapezoidal cantilever structure. It is also considered that the nonlinear piezoelectric coefficient $\left(g_{31}\right)$ might increase the output power at that region of PZT thin film. More accurate quantitative analysis of these results is under investigation.

The strain profiles along the length of the cantilever for both cantilevers were plotted in the Fig. 5. Three electrodes were located in the range of $0 \sim 0.2,0.4 \sim 0.6$, and $0.8 \sim 1.0$. While the rectangular cantilever showed a linear decrease, the trapezoidal cantilever had improved strain distribution along the length of the cantilever. All electrodes were connected together, and their output power was re-measured for both cantilevers to compare the total powers without normalization, as shown in Fig. 6. The maximum power was obtained at the resistive load of $7 \mathrm{k} \Omega$ for both cantilevers. The output power from the trapezoidal cantilever shows $39 \%$ higher than that of the rectangular cantilever.

\section{Conclusions}

The local strain and output power as a function of cantilever geometry were investigated and compared to values obtained from simulation and experiments by using a micromachined PZT energy harvester. The strain distribution with higher values along the cantilever beam was verified on the trapezoid-shaped cantilever as compared to the rectangular shape. The generated power from individual electrodes located near the anchor, middle, and mass has good agreement with the strain distribution. The results show that modulation of strain distribution can be an effective way to improve the output power in MEMS-scale devices. 


\section{Acknowledgment}

Authors appreciatively acknowledge the financial support provided by the National Science Foundation (NSF-DMR-0605270), and Auburn University Detection and Food Safety Center funded by USDA-CSREES.

\section{References}

1. R. Roundy, L. Frechette, P. Wright, and J. Rabaey, Power sources for wireless sensor networks Wirelss sensor Networks. EWSN Proc. 1-17 (2004).

2. P. D. Mitcheson, T. C. Green, E. M. Teatman, and A. S. Holmes, Architectures for vibration-driven micropower generators. J. Micro. Sys. 13, 429-440 (2004).

3. R. N. Torah, S. P. Beeby, M. J. Tudor, T. O'Donnel, and S. Roy, Kinetic energy harvesting using micro scale electromagnetic generators. in 17th MME. 2006; Southampton, UK.

4. S. Roundy, P. K. Wright, and J. Rabaey, A study of low level vibrations as a power source for wireless sensor nodes. Comput Commun. 26, 1131-1144 (2003).

5. D. Shen, S. Y. Choe, and D. J. Kim, Analysis of piezoelectric materials for energy harvesting devices under high-g vibrations. JJAP 46(10A), 6755-6760 (2007).

6. R. Roundy, E. S. Leland, J. Baker, E. Carleton, E. Reilly, E. Lai, B. Otis, J. M. Rabaey, P. K. Wright, and V. Sundararajan, Improving power output for vibration-based energy scavengers. IEEE Pervasive Comput. 4(1), 28-36 (2005).

7. D. Shen, J. H. Park, J. Ajitsaria, S. Y. Choe, H. C. Wikle, and D. Kim, Design, fabrication iand evaluation of a mems pzt cantilever with an integrated si proof mass for vibration energy harvesting. J. Micromech. Microeng. 18(5), 055017 (2008).

8. M. Marzencki, Y. Ammar, and S. Basrour, Integrated power harvesting system including a mems generator and a power management circuit. Proc. Int. Conf. on Solid-State Sensors, Actuators and Microsystems 887-890 (2007).

9. H. B. Fang, J. Q. Liu, Z. Y. Xu, L. Dong, L. Wang, D. Chen, B. C. Cai, Y. Liu, Fabrication an dperformance of mems-based piezoelectric power generator for vibration energy harvesting. Microelect. J. 37, 1280-1284 (2006).

10. Y. B. Jeon, R. Sood, J. H. Jeong, and S. G. Kim, MEMS power generator with transverse mode thin film pzt. Sen and Act. A 122, 16-22 (2005).

11. J. Baker, S. Roundy, and P. Wright, Alternative geometries for increasing power density in vibration energy scavenging for wireless sensor networks. in 3rd International Energy Conversion Engineering Conference. 2005; San Freansico: AIAA.

12. Y. Tadesse, S. Zhang, and S. Priya, Mulimodal energy harvesting system: Piezoelectric and electromagnetic. J. Int. Mat. Sys. Str. 20, 625-632 (2009). 